\title{
Free radicals in skin and muscle: damaging agents or signals for adaptation?
}

\author{
Malcom J. Jackson \\ Department of Medicine, University of Liverpool, Liverpool L69 3GA, UK
}

\begin{abstract}
Much of the current literature regarding the biological effects of antioxidant nutrients has concentrated on their potential role in inhibiting or preventing tissue damage induced by free radical species produced during metabolism. Recent findings indicate that antioxidants may also have more subtle roles, regulating changes in gene expression induced by oxidizing free radical species. There is increasing evidence that free radicals act as signals for cell adaptation in a variety of cell types and the nature of the mechanisms by which free radical species influence gene expression is the subject of much current research. Processes such as these may be particularly important in tissues regularly exposed to varying amounts of oxidative stress as part of their normal physiological functions. Examples of such tissues include skin exposed to u.v. light and skeletal muscle subjected to repeated bouts of exercise.
\end{abstract}

Antioxidants: Oxidative stress: Free radicals: Skeletal muscle: Skin

In recent years free radical species have been implicated in the pathogenesis of a number of different disorders, although therapeutic trials of agents designed to reduce the activity of these free radicals have generally been unrewarding (Halliwell \& Gutteridge, 1989). Initial studies examined the possibility that free radicals were the major cause of tissue degeneration in a number of different situations, but it is becoming increasingly clear that free radicals are more likely to play discrete, but crucial, roles in complex pathophysiological processes where there may be no obvious direct link between the pathological consequences and a rise in cellular oxidative stress. One example of this type of mechanism is the degenerative process seen following prolonged tissue ischaemia and subsequent reperfusion. In this situation antioxidant supplementation may offer some protection against tissue damage, but blockers of cellular $\mathrm{Ca}$ uptake and inhibitors of neutrophil activation or sequestration have equivalent protective effects (Bushell et al. 1996).

\section{Cellular adaptation to oxidative stress}

Cells respond to increased oxidative stress by adaptive changes in the expression of a variety of proteins involved in maintenance of cellular integrity. This response has been extensively studied in model systems by Davies and co-workers (Wiese et al. 1995), who have shown that mammalian cells respond to varying levels of oxidative stress by variations in the rate of cell growth, changes in the length of the cell cycle, and marked adaptive responses in resistance to oxidative stress. The process has not been fully explored in other systems, although analogous changes must occur and inevitably differ in other cell types, such as those which are unable to divide and enter the cell cycle. These responses include an up-regulation of the activity of antioxidant enzymes such as catalase (EC 1.11.1.6) and superoxide dismutase (EC 1.15.1.1), increased activity of DNA repair enzymes, and expression of cytoprotective proteins such as heat-shock proteins.

These observations raise the possibility that cells have sought to utilize free radical species as agents to signal the onset of an adaptive response of the cell to certain physiological stresses. Free radicals are produced during exposure of cells to many physiological stresses which in moderate amounts are known to induce adaptive responses, but if they are continued or magnified would lead to tissue damage (Fig. 1). Examples of these processes are the effects of exercise on skeletal muscle and the effects of u.v. light on skin.

\section{Exercising skeletal muscle: free radical generation and adaptation}

Skeletal muscle is repeatedly subjected to bouts of oxidative stress during exercise. Considerable data now indicates that 


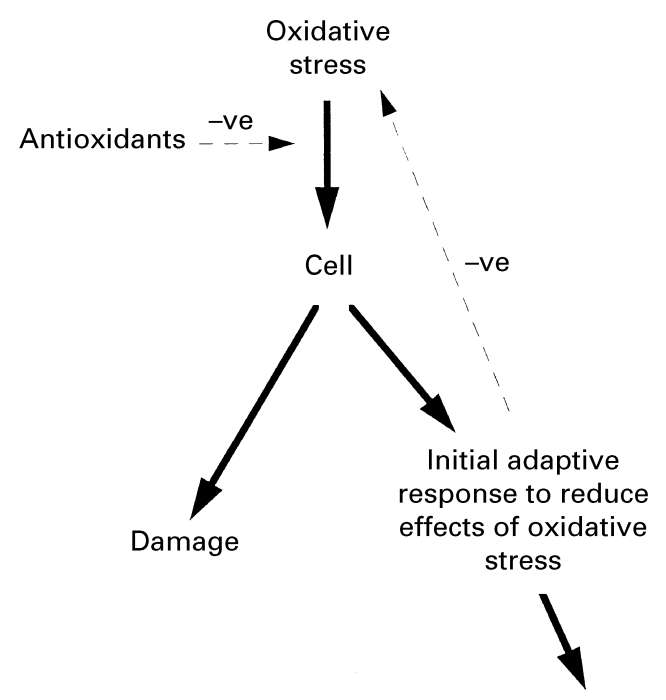

Secondary adaptation to prevent occurrence of oxidative stress

Fig. 1. Schematic diagram illustrating the concept that free radicals can stimulate both damage to cells and adaptation even in situations where overt cellular damage does not occur. The initial rapid adaptation appears to be designed to reduce the deleterious effects of the oxidative stress (e.g. elevations in the activity of antioxidant enzymes such as superoxide dismutase $(E C$ 1.15.1.1) and catalase (EC 1.11.1.6)), while later changes seem designed to prevent the stress occurring (e.g. adaptations in muscle oxidative capacity etc.). -ve, Negative.

aerobic contractile activity is associated with an increase in free radical production in skeletal muscle (Davies et al. 1982; Jackson et al. 1985). This increased production arises since a proportion of the molecular oxygen used in normal respiration undergoes one-electron reduction to produce superoxide radicals (Boveris et al. 1972), the production of which increases with the large increase in $\mathrm{O}_{2}$ flux in muscle mitochondria during exercise. This process leads to release of superoxide and $\mathrm{H}_{2} \mathrm{O}_{2}$ by the muscle cell and the local formation of hydroxyl radicals (O'Neill et al. 1996; see Fig. 2). Much work has been undertaken to examine the possibility that this free radical production is the cause of exercise-induced muscle damage, although the evidence remains inconclusive (for review, see Jackson, 1996).

There is considerable evidence that muscle cells adapt to this increased free radical activity to reduce the risk of free radical damage to the tissue. Thus, exercise training has been shown to increase the activity of several antioxidant enzymes such as superoxide dismutase and catalase in muscle, and recent findings indicate an increase in muscle heat-shock protein content following exercise (Salo et al. 1991). It is now recognized that these adaptations can protect skeletal muscle against further bouts of (normally) damaging contractile activity (McArdle et al. 1997). In the latter studies mice underwent non-damaging exercise for 15 min, and $4 \mathrm{~h}$ later (when tissue heat-shock protein content was elevated) the pre-exercised muscle was subjected to a normally-damaging exercise protocol. This procedure induced a substantial resistance to contraction-induced damage in the pre-exercised muscles.

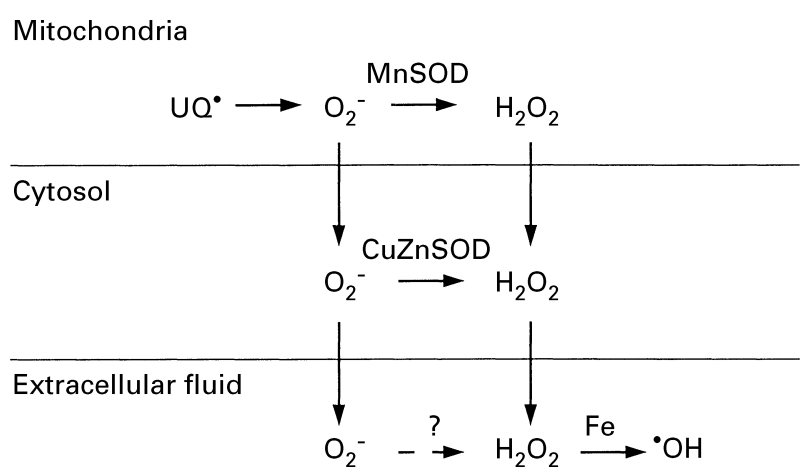

Fig. 2. Schematic diagram of kinetics of free radical generation from mitochondria of contracting skeletal muscle. The proposed mechanism explains the findings of an elevated free radical signal, derived from ubiquinone (UQ), which can be seen on electron spin resonance examination of contracting muscle, the detection of superoxide release from contracting muscle and the finding of irondependent generation of hydroxyl radicals in the venous effluent from contracting muscle (for references, see p. 674). Mn, CuZn SOD, manganese and copper, zinc superoxide dismutase (EC 1.15.1.1); $\cdot \mathrm{OH}$, hydroxyl radical.

There is increasing evidence, therefore, that exerciseinduced oxidative stress induces an adaptive response in skeletal muscle which serves to protect the tissue against further stress. The impact of antioxidant supplementation on this process is not clear. One mechanism by which this adaptation to increased free radical activity might occur is by influencing the activity of various transcription factors. In essence, free radicals can influence the ability of transcription factors, such as nuclear factor- $\mathrm{\kappa B}$, activation protein-1 and heat-shock factor-1, to bind to their respective sites within the promoter region of specific genes. This process appears to be mediated by oxidation of redoxsensitive cysteines within the binding region (Stortz \& Polla, 1996; Jackson et al. 1998).

\section{Exposure of skin to u.v.B: free radical generation and adaptation}

Excessive exposure of skin to u.v. light can result in either acute or chronic damage (Miyachi, 1987). Short-term overexposure to u.v. causes acute damage, known as erythema or sunburn, whilst chronic overexposure can lead to increased risk of skin cancer and premature photoageing (Kligman \& Kligman, 1986). Recently, concern has been expressed that human subjects are becoming exposed to higher doses of u.v. due to depletion of ozone in the atmosphere (Frederick, 1993). The u.v. light is composed of u.v.A $(320-400 \mathrm{~nm})$, u.v.B (280-320 nm) and u.v.C (200-280 nm), but only u.v.A and u.v.B reach the earth's surface and are of physiological significance (Frederick, 1993; Tyrell, 1996). Exposure of cutaneous tissue in vitro and in vivo is known to generate free radicals and other reactive oxygen species (Black, 1987), resulting in cellular oxidative stress (Darr \& Fridovich, 1994), which has been claimed to contribute to the accelerated ageing process (Harman, 1981).

There has been considerable discussion concerning the potential role of oxidative stress in the deleterious effects of 
u.v. on human skin. Most interest in this area has concerned the effects of u.v.A in generating free radical and reactive oxygen species in skin cells, but our findings now indicate that u.v.B stimulates superoxide release from human skin fibroblasts. This release was found to be dose dependant and led to a rise in cell lipid peroxidation (shown by an increase in malonaldehyde content) and loss of cell viability over a longer time course (Jones et al. 1998). The mechanism by which u.v.B induced this rise in superoxide release is unknown, although both generation of superoxide by interaction of u.v. with melanin (Crippa \& Mazzini, 1983) and u.v.B stimulation of the mitochondrial electron transport chain with consequent release of increased superoxide anions (Masaki \& Sakurai, 1997) have been proposed.

Skin is known to respond to u.v.B by up-regulation of a number of protective systems. Pathak \& Stratton (1968) initially suggested that some responses to u.v. exposure may be mediated by free radicals, and subsequently other workers have supported this suggestion (Shindo et al. 1994; Chen et al. 1995; Masaki et al. 1995; Jurkiewicz \& Buettner, 1996).

Our findings indicate that human skin fibroblast cells respond to u.v.B by a transient rapid up-regulation of the activity of the antioxidant enzymes superoxide dismutase and catalase (Jones et al. 1998), and a slower but more marked increase in cellular GSH content (SA Jones, F McArdle, CIA Jack \& MJ Jackson, unpublished results). Such changes appear to be aimed at protecting the cells against further exposure to u.v. light. The mechanisms underlying these adaptive responses is currently unclear. The u.v.B-induced changes in superoxide dismutase and catalase activity are suppressed by supplementation of the cells with the water-soluble vitamin E analogue, Trolox (Sigma Chemical Co., Poole, Dorset, UK), although they are not reproducibly inhibited by other antioxidants such as ascorbate (Jones et al. 1998). These findings therefore imply that the u.v.B-induced adaptive changes are mediated by a free radical species which can be scavenged by Trolox.

\section{Conclusions}

The studies reviewed here indicate that free radicals mediate some of the adaptive changes which occur following certain physiological stresses in tissues, such as muscle following exercise and skin following u.v.B exposure. These findings also raise a number of important questions concerning the possible role of free radical species as signals for wider adaptive responses in these and other tissues, and question the approach to protection of tissues which involves advocation of widespread supplementation with antioxidant nutrients. It is entirely feasible, although not proven, that those adaptations to stress mediated by free radicals play an important role in maintaining cell viability in tissues routinely subjected to repeated stresses (e.g. muscle following exercise, etc.) and that increased consumption of some antioxidant nutrients might interfere with these necessary adaptive processes. Only further research in this area will determine the validity of such concerns.

\section{Acknowledgements}

The author's research programme receives financial support from the Medical Research Council, Biotechnology and Biological Sciences Research Council, Ministry of Agriculture, Fisheries and Foods and the Wellcome Trust. The author would also like to thank the large number of collaborators and colleagues who have contributed to the development of the ideas outlined here.

\section{References}

Black HS (1987) Potential involvement of free radical reactions in ultraviolet light-mediated cutaneous damage. Photochemistry and Photobiology 46, 213-221.

Boveris A, Oshire N \& Chance B (1972) Cellular production of hydrogen peroxide. Biochemical Journal 128, 617-630.

Bushell AJ, Klenerman L, Davies HM, Grierson I \& Jackson MJ (1996) Ischaemia-reperfusion induced muscle damage: Protective effects of corticosteriods and antioxidants in rabbits. Acta Orthopaedica Scandinavica 67, 393-398.

Chen T, Li W, Schultz PJ, Furst A \& Chien PA (1995) Induction of peroxisome proliferation and increase of catalase activity in yeast, candida-albicans, by cadmium. Biological Trace Element Research 50, 125-133.

Crippa PR \& Mazzini L (1983) Involvement of superoxide ions in the oxidation of NADH by melanins. Physiological Chemistry and Physics and Medical NMR 15, 51-56.

Darr D \& Fridovich I (1994) Free radicals in cutaneous biology. Journal of Investigative Dermatology 102, 671-675.

Davies KJA, Quintanilla AT, Brooks GA \& Packer L (1982) Free radicals and tissue damage produced by exercise. Biochemical and Biophysical Research Communications 107, 1198-1205.

Frederick JE (1993) Ultraviolet sunlight reaching the earth's surface: a review of recent research. Photochemistry and Photobiology 57, 175-178.

Halliwell B \& Gutteridge JMC (1989) Free Radical Biology and Medicine. Oxford: Oxford University Press.

Harman D (1981) The aging process. Proceedings of the National Academy of Sciences USA 78, 7424-7128.

Jackson MJ (1996) Oxygen-derived radical production and muscle damage during running exercise. In Human Muscular Function During Dynamic Exercise, pp. 121-133 [P Marconnet, B Saltin, P Komi and J Poortmans, editors]. Basel: Karger.

Jackson MJ, McArdle A \& McArdle F (1998) Antioxidant micronutrients and gene expression. Proceedings of the Nutrition Society 57, 301-305.

Jackson MJ, Symons MCR \& Edwards RHT (1985) Electron spin resonance studies of intact mammalian skeletal muscle. Biochimica et Biophysica Acta 847, 185-190.

Jones S, Jack CIA \& Jackson MJ (1998) Differential effects of supplementation with vitamin $\mathrm{E}$ analogues on antioxidant enzyme expression in human skin fibroblasts exposed to ultraviolet B. Proceedings of the Nutrition Society 57, 34A.

Jurkiewicz BA \& Buettner GR (1996) EPR detection of free radicals in UV-irradiated skin: Mouse versus human. Photochemistry and Photobiology 64, 918-922.

Kligman LH \& Kligman AM (1986) The nature of photoaging: Its prevention and repair. Photodermatology 3, 215-227.

McArdle A, McArdle C \& Jackson MJ (1997) Stress proteins and protection of skeletal muscle against contraction-induced skeletal muscle damage in anaesthetised mice. Journal of Physiology 499, 9P.

Masaki H, Atsumi T \& Sakurai H (1995) Detection of hydrogen peroxide and hyroxyl radicals in murine skin fibroblasts under 
UVB irradiation. Biochemical and Biophysical Research Communications 206, 474-479.

Masaki H \& Sakurai H (1997) Increased generation of hydrogen peroxide possibly from mitochondrial respiratory chain after UVB irradiation of murine fibroblasts. Journal of Dermatological Science 14, 207-216.

Miyachi Y (1987) Reactive oxygen species in photodermatology. The Biological Role of Reactive Oxygen Species in Skin, pp. 37-41 [O Hayashi, S Imamura and Y Miyachi, editors]. New York: Elsevier Science.

O’Neill CA, Stebbins CL, Bonigut S, Halliwell B \& Longhurst JC (1996) Production of hydroxyl radicals in contracting skeletal muscle of cats. Journal of Applied Physiology 81, 1197-1206.

Pathak MA \& Stratton K (1968) Free radicals in human skin before and after exposure to light. Archives of Biochemistry and Biophysics 123, 468-476.
Salo DC, Donovan CM \& Davies KJA (1991) HSP70 and other possible heat shock or oxidative proteins are induced in skeletal muscle, heart and liver during exercise. Free Radical Biology and Medicine 11, 239-246.

Shindo Y, Witt E, Han D \& Packer L (1994) Dose-response effects of acute ultraviolet irradiation on antioxidants and molecular markers of oxidation in murine epidermis and dermis. Journal of Investigative Dermatology 102, 470-475.

Stortz G \& Polla BS (1996) Transcriptional regulators of oxidative stress-inducible genes in prokaryotes and eukaryotes. In StressInducible Cellular Responses, pp. 239-254 [U Fiege, RI Morimoto, I Yahara and BS Polla, editors]. Basel: Birckhauser Verlag.

Tyrell RM (1996) Ultraviolet radiation and free radical damage to the skin. Biochemical Society Transactions 61, 47-53.

Wiese AG, Pacifici RE \& Davies KG (1995) Transient adaptation to oxidative stress in mammalian cells. Archives of Biochemistry and Biophysics 318, 231-240. 